\title{
Original and Scientific Contributions to Cyber - Mechatronics Systems Development for Industrial, Economical and Societal Environments
}

\author{
Gheorghe Gheorghe, Constantin Anghel, and Ilie Iulian
}

\begin{abstract}
The scientific paper presenting original contributions in developing specialized domain "Cyber-mix mechatronics" through new and advanced cyber-mix mechatronics systems with applications in many industrial, economical and societal environments.

The scientific work generally included physical world with the virtual world systems or mechatronic engineering mix with the Internet, assembling into mix mechatronics cyber systems for measurement and control processes and processing, energy networks, microsatellites network, and so on.
\end{abstract}

Index Terms-Cyber-mix mechatronics systems, advanced technologies, physical systems.

\section{INTRODUCTION}

This scientific work responds to "challenge of the European strategic paradigm" creation and development of new concepts and new intelligent systems multiplied in industrial value chains and who require training, combining and merging of the highest competence and innovative solutions, especially the most mechatronic technologies and cyber-mechatronic solutions that integrate advanced skills and high-tech Innovation Technology \& Communication for efficiency and eco-innovation of products, more advanced and smarter.

Thus, the complex mechatronic systems and cyber-mechatronic based on science and engineering mechatronics and cyber-mechatronics integrating in architecture of sensors and intelligent network of sensors, actuators and transfer processes and processing data, processed and synthesized, interacting with the physical world and with the virtual world and supports real-time, guaranteed performance and safety in all applications in industry, economy and society.

These cyber-mechatronics systems [1] facilitate behaviour as a whole all mechatronic and cyber-mechatronic elements, all elements of the calculation, control, remote control, sensing and remote sensing, assembled in smart grids and integrated action safe and interoperable, they are multiplicative, and areadaptive for the future.

Multi applicability and multi adaptivity include matrix

Manuscript received February 1, 2017; revised April 23, 2017.

Gheorghe Gheorghe is with Sos. Pantelimon, 6-8, 021631, Bucharest, Romania (e-mail: geocefin@yahoo.com).

Constantin Anghel and Ilie Iulian are with Str. Lt. Staicu Ion, 35, 130105, Targoviste, Romania (e-mail: anghel.constantinl@incdmtm.ro, ciulian.ilie@incdmtm.ro). flexible architecture of intelligent systems, through complex structures of sensors and actuators that transmit information on $4 \mathrm{G}$ devices and smart handling, storing and transferred to entities / monitoring centres and remote monitoring for command and management, respectively remote control.

These systems will be built multiplicative and multi adaptive form of entity "black box" that will integrate hardware and software for the capture, modelling and data communication to and from the command centre.

Systems architecture will be a modular, with the possibility of integrating other smart devices type "add-on" special or dedicated to specific activities.

The types of data captured will be processed, normalized and standardized system formats that provide interoperability, platforms that provide real-time monitoring. Between mother intelligent systems and command centre, there is a communication protocol through a secure exchange of information, record data in real time and display them. Command centre is operated by WEB platform, issues real-time data, places and commands such as $\mathrm{ON} / \mathrm{OFF}$ is being transmitted sequentially to mother systems and on smart devices targeted by these commands.

\section{Complex ApPlication of Mechatronics AND CYBER-MECHATRONICS CONCEPT IN INTELLIGENT COMPLEX SYSTEMS}

The application of mechatronics, cyber-mechatronics and cyber-mix mechatronics complex concept, to the design and implementation of intelligent cyber-mechatronics systems will be varied and diversified for many industrial, economical and societal environments, as follows:

-Cyber-mechatronic system, robot fo dimensional control with remote monitoring and remote control (Fig. 1)

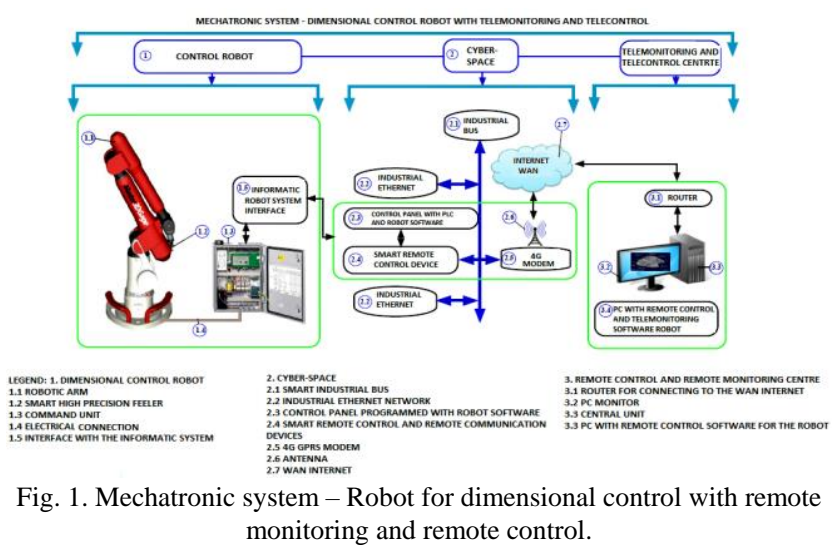


mechatronics systems with remote monitoring and remote control (Fig. 2)

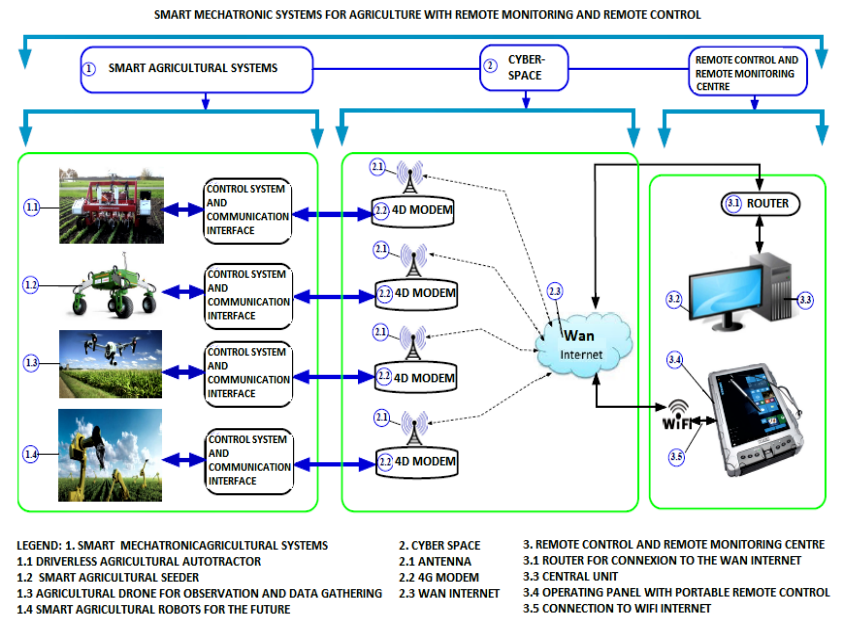

Fig. 2. Agricultural mechatronics systems with telemonitoring and remote control.

-Cyber-mechatronic system, intelligent network of renewable energy sources with remote monitoring and remote control (Fig. 3)

SMART NETWORK OF SOURCES OF RENEWABLE ENERGY WITH TELEMONITORING AND TELECONTRO

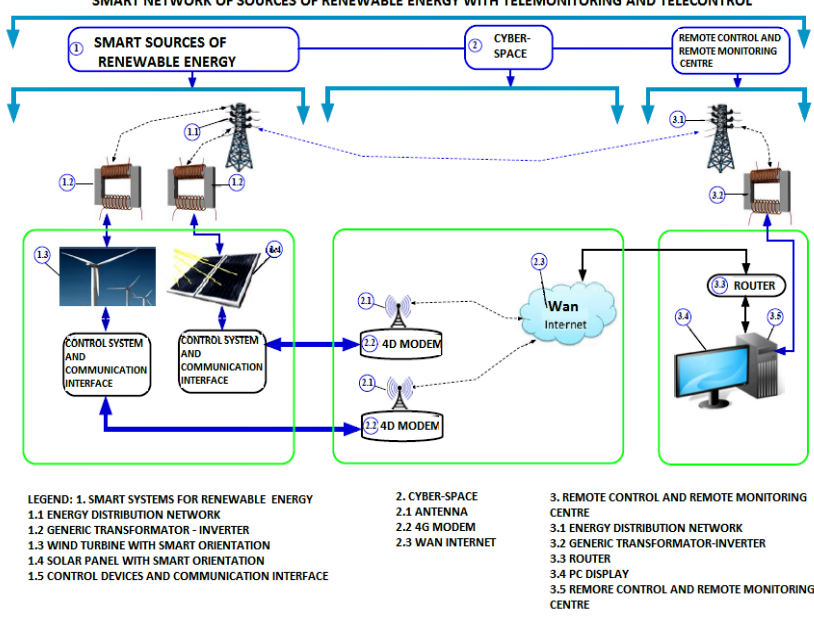

Fig. 3. Mechatronic system, intelligent network of renewable energy sources with telemonitoring and remote control.

-Cyber-mechatronic system, remote sensing systems with the laser beam for a network of microsatellites (Fig. 4)

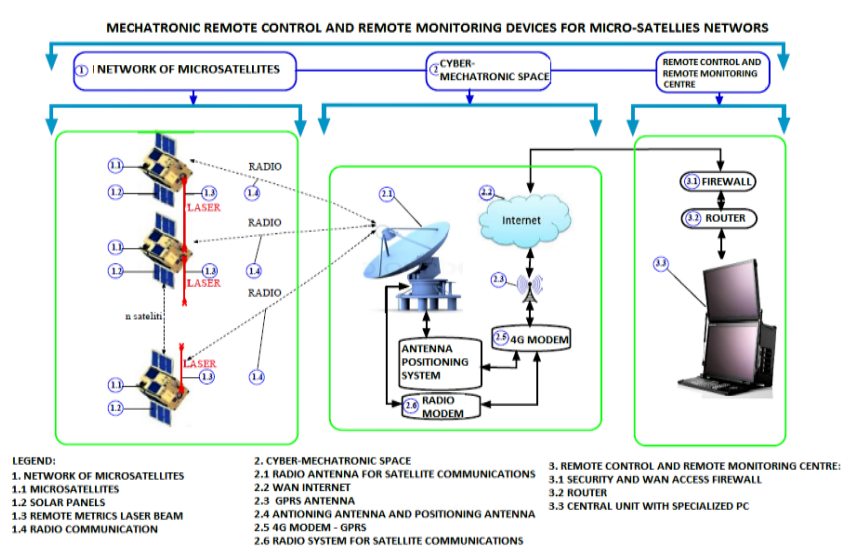

Fig. 4. Remote sensing mechatronic system with the laser beam for a network of microsatellites.

-Cyber-mechatronic system, intelligent equipment of remote monitoring and remote control for Earth observation rooms of cosmic waste (Fig. 5)

SMART REMOTE CONIROL AND REMOTE MINITORING MECHATRONIC SYSTEM FOR TERRESTRIAL CONSERVATION CHAMBER FOR COSMIC DEBRIS

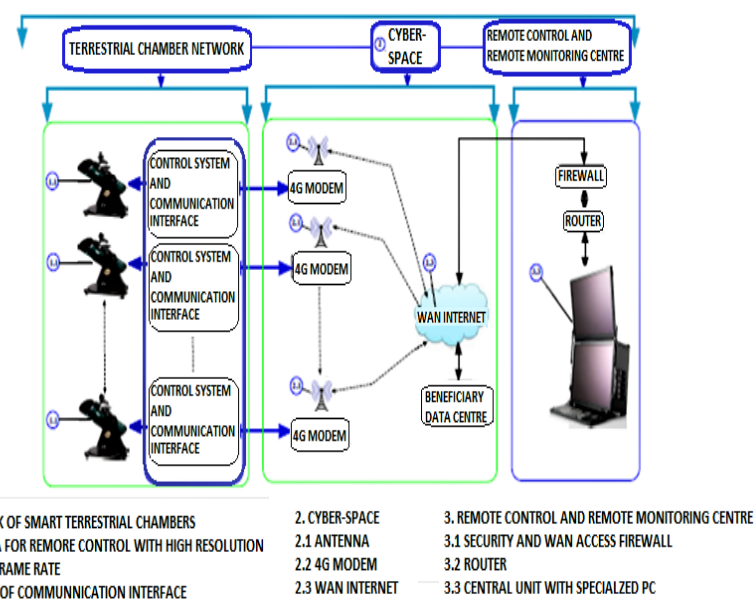

Fig. 5. Cyber-mechatronic system, intelligent equipment of remote monitoring and remote control for Earth observation rooms of cosmic waste.

\section{ORIGINAL SCIENTIFIC AND TECHNICAL CONTRIBUTIONS TO THE CONCEPTION AND DEVELOPMENT OF CYBER-MECHATRONICS SYSTEMS}

\section{A. For Cyber-Mechatronic System Operation, Control Robot with Remote Monitoring and Remote Control}

At international level were developed various equipment, computer controlled, mechatronics robotics and virtual systems.

Mechatronics robots [2], [3] are used in many fields, intervention in hostile environments, in surgery, in the military, the remote control of industrial processes (such as integrated dimensional control), etc.

The system shown is a mechatronic robotic dimensional control system, integrated and controlled remotely and through remote monitoring and Remote control Centre.

Mechatronic robotic positioning system, guide and manipulate high precision stylus tool, as programmed by the command centre.

As a rule, the action of robotic arm equipped with high precision feeler gauge is much better achieved by the robot compared to other mechatronic systems with manual measurement.

Based on the concept of "artificial intelligence" [4]-[8] can appreciate that mechatronics control robots must satisfy two main components, namely logical component and mathematical component. These are already achieved when the computing systems have been developed for high performance.

When testing the communication network to be used in the management and / or monitoring process are taken into account the delays mean the probability of error / data packet loss. If these time delays are much smaller than the sampling period required, the process can be conducted by incorporating a delay block. If the delays are comparable to the sampling period then is recommended a structure that uses a Smith preacher (closed loop control system with dead time compensation). time compensation). 
After experiments performed, could group into three distinct situations remote automatic control processes:

Case 1: predictive control for automated systems with delays.

Case 2: adaptive predictive control using a calculated delay a-posteriori that, based on the experimental values are calculated an average value that is used in automatic control alghorithm.

Case 3: adaptive predictive control using an estimated variable delay a-priori: based on experimental values was estimated delay time to the next sampling period.

Further, it shows subsystems of mechatronic components of mechatronic robotic assembly system: -

Mechatronic subsystem - control robot (Fig. 6)

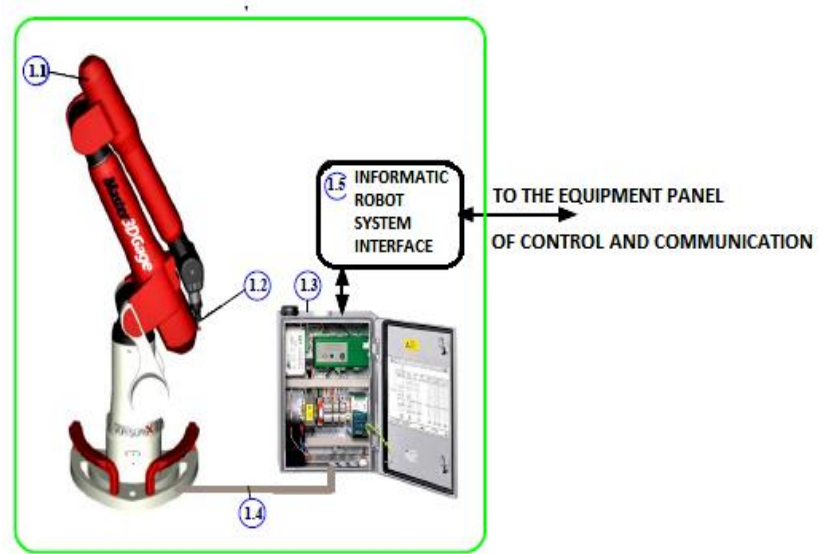

1. DIMENSIONAL CONTROL ROBOT

1.1 ROBOTIC ARM

1.2 SMART HIGH PRECISION FEELER

1.3 COMMAND UNIT

1.4 ELECTRICAL CONNECTIONS

1.5 INTERFACE WITH THE INFORMATIC

SYSTEM

Fig. 6. Mechatronic subsystem - control robot.

Mechatronic subsystem - cybernetic space (Fig. 7)

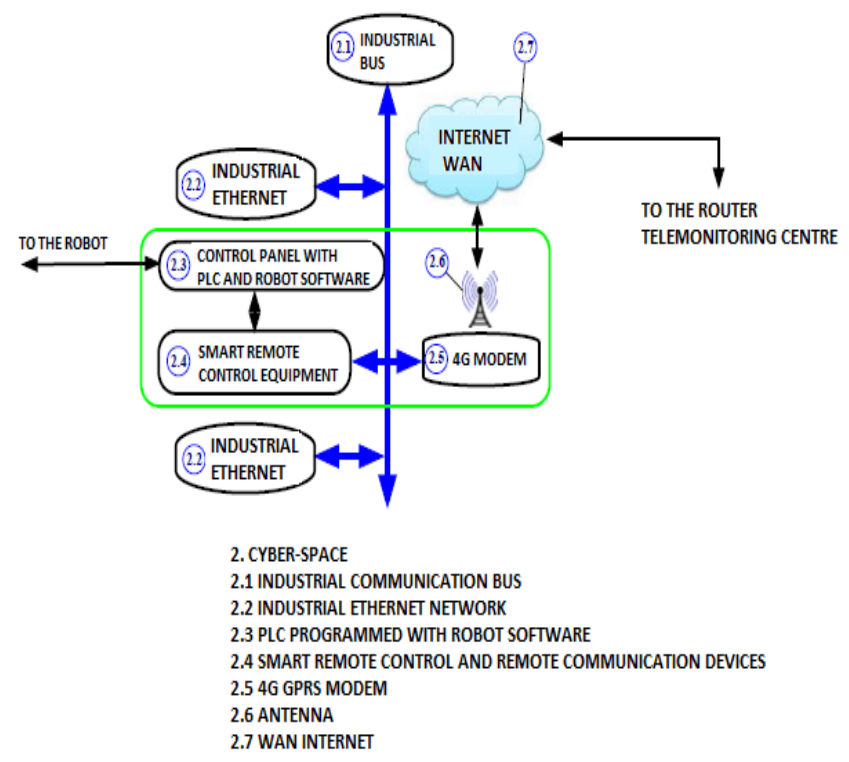

Fig. 7. Mechatronic subsystem - cybernetic space.

Mechatronic subsystem - centre of remote monitoring and remote control (Fig. 8)

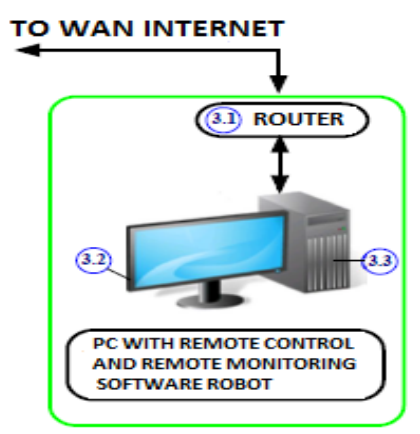

3. REMOTE CONTROL AND REMOTE MONITORING CENTRE 3.1 ROUTER FOR CONNECTING TO WAN INTERNET

3.2 PC DISPLAY

3.3 CENTRAL UNIT 3.4UPC WITH REMOTE CONTROL ROBOT
SOFTWARE

Fig. 8. Mechatronic subsystem - centre of remote monitoring and remote control.

Whether $\mathrm{O}_{0}\left(\mathrm{X}_{0} \mathrm{Y}_{0} \mathrm{Z}_{0}\right)$ the reference system linked to the base of the robot and $\mathrm{W}_{0}\left(\mathrm{X}_{\mathrm{w}} \mathrm{Y}_{\mathrm{w}} \mathrm{Z}_{\mathrm{w}}\right)$ the coordinate system with the origin in $\mathrm{W}_{0}$ (the origin of gripping system jointly with the feeler gauge). In Fig. 8 shows the positions of the two reference systems, respectively the point $\mathrm{O}_{0}$ and $\mathrm{W}_{0}$ positions.

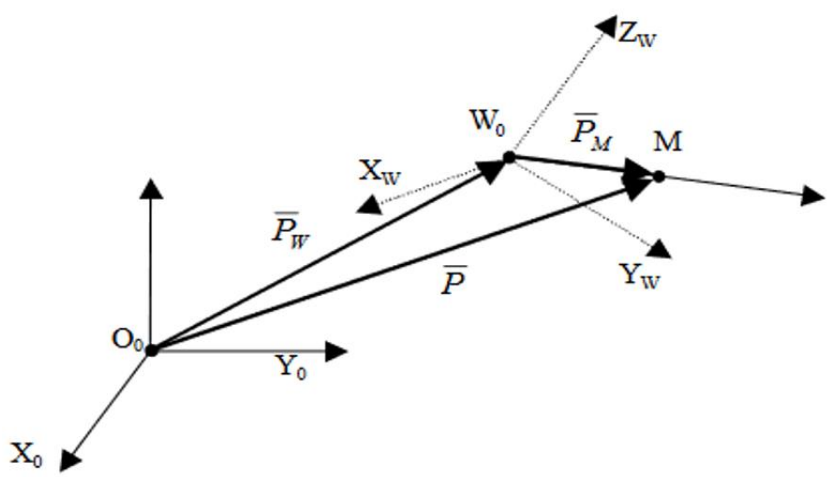

Fig. 9. The coordinate systems of the robot and position vector $\mathrm{P}_{\mathrm{w}}$

Based on Fig. 9 can make notations:

$\vec{P}=\left[p_{x}, p_{y}, p_{z}\right]$ position vector of characteristic point $\mathrm{M}$ in the coordinate system $\mathrm{O}_{0}\left(\mathrm{X}_{0} \mathrm{Y}_{0} \mathrm{Z}_{0}\right)$.

$\overrightarrow{P_{W}}=\left[p_{w x}, p_{w y s}, p_{w z}\right]$ position vector of characteristic point $\mathrm{M}$ in the coordinate system $\mathrm{O}_{0}\left(\mathrm{X}_{0} \mathrm{Y}_{0} \mathrm{Z}_{0}\right)$.

$\overrightarrow{P_{M}}=\left[p_{m x}, p_{m y}, p_{m z}\right]$ position vector of characteristic point $\mathrm{M}$ in the coordinate system attached to gripping device jointly with the feeler gauge having the origin in $\mathrm{W}_{0}$ point.

The matrix of location $\mathrm{T}$ (specific term regarding a homogeneous transformation expressing the relative position of the two solids), through are expressing the location of final effector of a mechatronic robot, in East three-dimensional space

$$
T=\left[\begin{array}{cccc}
n_{x} & o_{x} & a_{x} & p_{x} \\
n_{y} & o_{y} & a_{y} & p_{y} \\
n_{z} & o_{z} & a_{z} & p_{z} \\
0 & 0 & 0 & 1
\end{array}\right]
$$

where: $p_{x}, p_{y}, p_{z}$ are components of position vector of 
characteristic point and $n_{x}, n_{y}, n_{z} o_{x,} o_{y}, o_{z} a_{x}, a_{y}, a_{z}$ are vector components $\vec{n}, \vec{o}$ and $\vec{a}$, expressing solidarity with the orientation of the gripping device jointly with the feeler gauge.

Further, it shows the logical diagram for the reverse kinematic analysis software for mechatronic robot with 5 degrees of freedom - Fig. 10 using the method by decomposition.

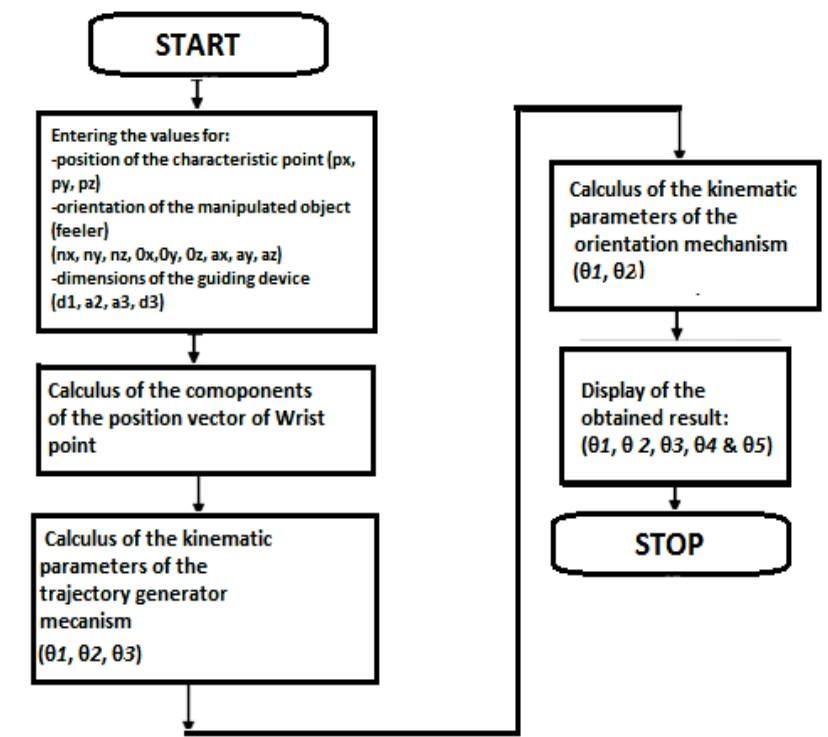

Fig. 10. The logical diagram for the reverse kinematic analysis software for mechatronic robot with 5 degrees of freedom.

Where it is considered that we know the position of characteristic point $(p x, p y, p z)$, the orientation of manipulated object ( $n x, n y, n z, o x, o y, o z, a x, a y, a z)$ toward the coordinate fixed system, linked to the robot base and the guiding device dimensions $(d 1, a 2, a 3, d 5)$ and aims to determine kinematic parameters $(\theta 1, \theta 2, \theta 3, \theta 4$ and $\theta 5)$ for the five kinematic rotation couples thus resulting from the logical diagram.

\section{Cyber SECURITY OF MeChATRONICS Robotics SYSTEMS AND COMMUNICATION PROCESSES}

Most hackers of computer networks, are malicious people who manage to get beyond a security barrier, intentionally destroying certain parts of the system or causing a malfunction.

The methods to ensure security systems have been standardized in ISO / IEC 27001: 2005 - Information Technology - Security Techniques - Information security management systems - Requirements, which has been joined to date, over 30 companies in Romania (according to certification body SRAC).

Possible methods of cyber-attack a network communication:

Sniffing (are some applications (or sometimes hardware) that do nothing more than

- Record the data packets that are circulating on network)

- Hacking (reverse engineering techniques (removal programs), with the scope to obtain knowledge

- or informatic facilities).
Social engineering (the art of getting information and unauthorized in different systems and / or locations).

Resuming whole philosophy of the security of information systems in a sentence can be said that "we should not be afraid of what will happen, but we need to know what will happen". Moreover, the principle of prudence and continuity of accounting combine perfectly with the security strategies of modern companies

To ensure optimum protection from various types of cyber-attacks using VPN technology (Virtual Private Network). They represent a new technique for wide area networks. Thus, a virtual private network, VPN, is a new type of system access for users who connect remotely. VPN systems allow remote users to connect to their network via the Internet. This connection is done by creating a "virtual" circuit from the computer to the network system; namely the link that appears private for other users, even if passing through the public system of Internet. All data and information circulating through the connection are encoded to not be read by void persons.

The idea is to connect private networks through a public network using a private protocol. In this way, communication with private resources is accessible only to users therein. The concept is called virtual private network, VPN; There are many protocols of this type, and basic public network is the Internet. VPN uses the Internet to connect with each other more LANs via a secure connection. VPN connections accomplishes this with two important technologies: creating tunnels and encryption. First, create a VPN over the Internet, "virtual circuit" between the two connected points. Then, using the method of creating tunnels to pack data into Internet protocol, TCP / IP, so that it can be easily transferred.

Encryption encodes information sent, so that only the recipient to whom is addressed to be able to decode and read. To implement a VPN are required: a firewall, router, proxy server, hardware and VPN software or whichever can provide a secure communication, but their combination is the best solution.

The local networks of users can be both wired and wireless. VPNs deployments are organized in Intranet VPN - virtual private network between offices and departments of the same institution;

\section{EXPERIMENTS AND TESTS IN INCDMTM LABORATORIES}

In INCDMTM were conducted preliminary tests in order to verify the technical and theoretical principle of cyber-mechatronic systems.

For this purpose, were designed specific software modules that enable configuration and testing of input vectors in space automation systems implemented automatic SIEMENS S7-300 family via PCs connected to the Intranet.

The main captures of suggestive screens are listed below for testing mechatronic generic cyber-systems.

Fig. 11 presents an example of a program that tests the communication between an S7-300 PLC and a PC at the centre of remote monitoring and remote control. 
Fig. 12 shows an example of a test monitoring program LOCAL input vectors.

Fig. 13 presents an example of a monitoring program to test vectors input Intranet.

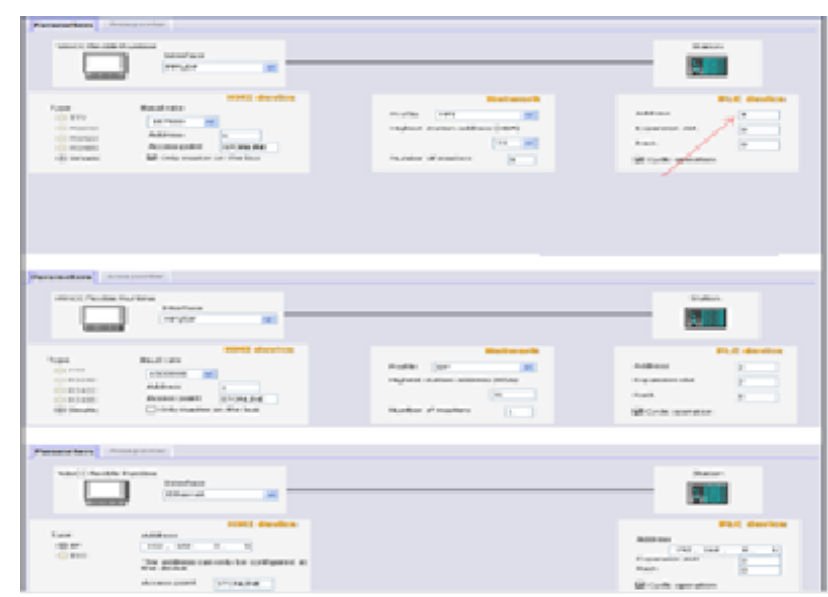

Fig. 11. Communication and configuration tests PLC S7-300.

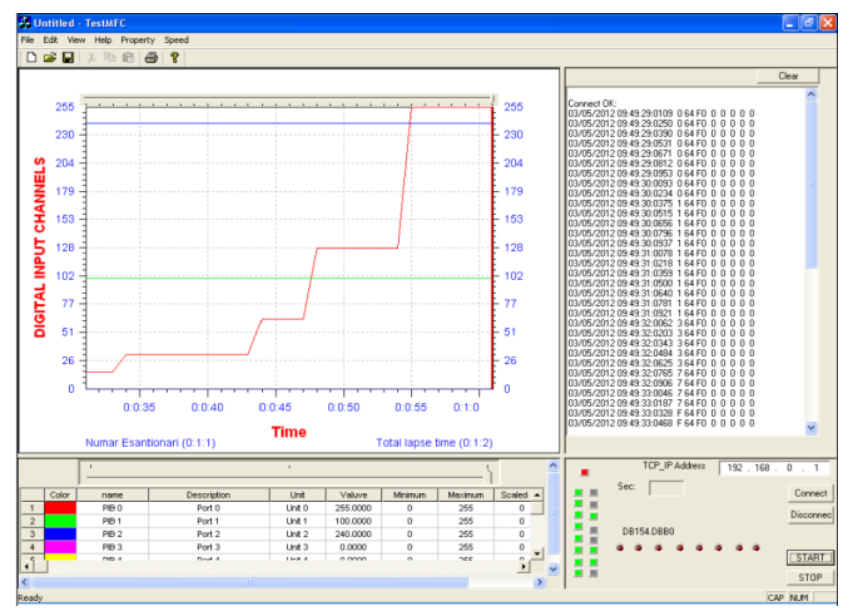

Fig. 12. Screen test LOCAL input monitoring vector.

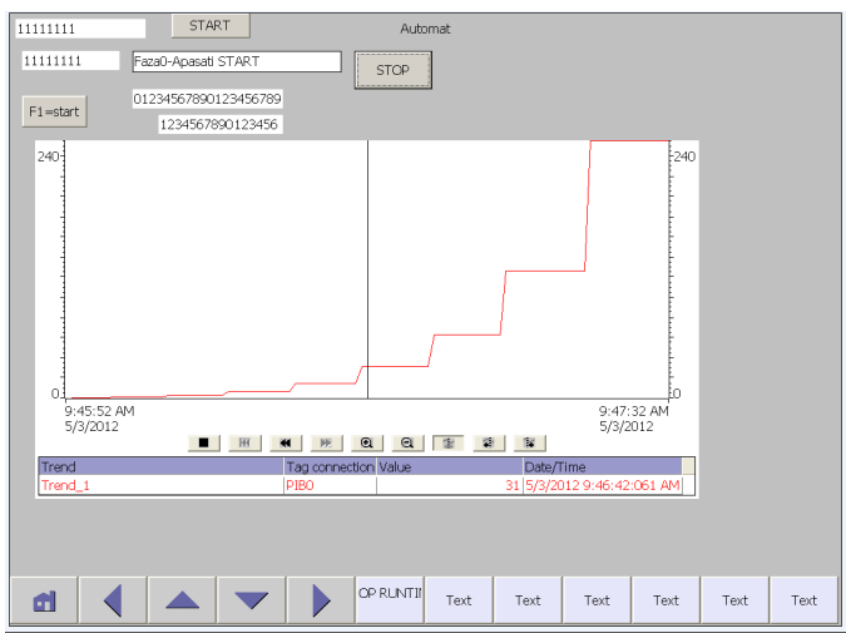

Fig. 13. Screen test INTRANET input monitoring vector.

The test results are in line with forecasts and expectations of theoretical studies conducted and represent the basis for the development of complex applications in order to remote monitoring and remote maintenance of mechatronic systems, applicable to a wide range of systems for measurement and control, delivered to different customers in the automotive industry and others industries (Renault-Dacia,

\section{TOPOLOVENI, TRANSGAZ).}

As a result of experiments have been grouped into three distinct situations for remote management processes.

Case 1: Predictive control for systems with delay.

Case 2: Predictive Adaptive Control using a-posteriori calculated delay: based on experimental values of the delays were calculated an average that is used by management algorithm.

Case 3: Predictive Adaptive Control using an estimated a-priori variable delay: based on experimental values was estimated delay time to the next sampling period.

\section{CONCLUSION}

The original and scientific contributions help at the development of cyber-mechatronic concept, by assembling the principles, methods and procedures applied to physical systems or mechatronic and virtual systems, namely the Internet.

Were designed in a wide range, many cyber-mechatronic systems for many industrial, economical and societal environments.

Thus, the authors have helped develop scientific knowledge and highly competitive, emphasizing the leading role and future of cyber-mix mechatronics engineering.

\section{REFERENCES}

[1] I. G. Gheorghe, A. Constantin, and I. Ilie, "Scientific evolution from mix - integrating mechatronics to cyber-intelligent mechatronics and to claytronics science," in Proc. of OPTIROB 2016.

[2] I. G. Gheorghe, A. Constantin, and I. Ilie, "Mechatronics and cyber mechatronics in intelligent applications from industry and society," in Proc. of OPTIROB 2016.

[3] W. Zesch et al., "Inertial drives for micro and nanorobots: Two novel mechanisms, proc of int. Symp. on microrobotics and micromechanical systems," pp. 80-88.

[4] V. Giurgiutiu and S. E. Lyshevski, "Micromechatronics: Modeling, analysis, and design with matlab," CRC Press, 2004, pp. 856.

[5] H. Guckel et al., "Spring constrained Linear Actuator with Large Throw," in Proc. of Int. Conf. on New Acuators, Bremen, pp. 52-55.

[6] B. S. Spencer, "Incorporating the sense of smell into patient and haptic surgical simulators," IEEE Trans. Inf. Technol. Biomed, vol. 10, no. 1, pp. 168-173, 2006.

[7] K. K. Shung and M. Zipparo, "Ultrasonic transducers and arrays. IEEE Eng. in Medicine and Biology Magazine," IEEE, vol. 15, no. 6, pp. 20-30.

[8] C. Nitu, C. D. Comeaga, and B. Gramescu, "Vibration control by use of piezoelectric patches, mechatronics," pp. 38-41.

[9] J. Machado, F. Soares, and L. Celina, "A virtual Workbench applied to Automation Students Response Analysis," in Proc. of Controlo 2014, Portugal.

[10] C. I. Rizescu, C. Udrea, and D. Rizescu, "Experimental setup for harmonic drive efficiency determination," Advanced Materials Research, vol. 463-464, pp. 1518-1521, 2012.

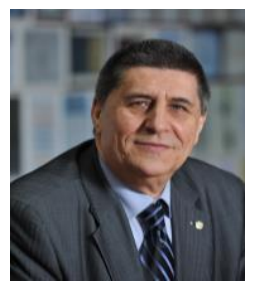

Gheorghe Gheorghe is an associate professor at the Faculty of Mechatronics Politehnica University of Bucharest, Valahia University of Targoviste an Titu Maiorescu University, Romania. He received her bachelor degree in precision mechanics from Politehnica University of Bucharest, doctoral degree at Politehnica University of Timisoara and Doctor Honoris Causa degree from Valahia University of Targoviste. His current research includes mechatronics and cyber-mechatronics systems, measurement technique, cyber - adaptronics systems. 


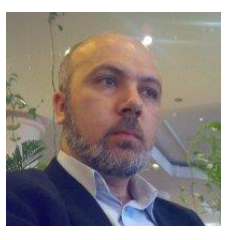

Anghel Constantin is currently doctoral student at Valahia University of Targoviste. Licence degree at Polytechnic University of Bucharest, Electronics,

Telecommunications and Information Technology Faculty, Master Degree at Polytechnic University of Bucharest, Faculty of Electronics, Telecommunications and Information Technology, Senior Researcher at National Institute of Research and Development for Mechatronics and Measurement Technique.

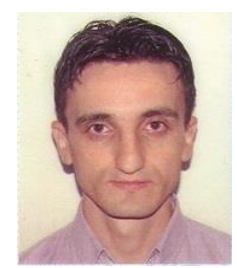

Ilie Iulian is currently octoral stuent at Valahia University of Targoviste. He graduated the Politehnica University of Bucharest with an Engineer diploma. Mr. Ilie has also a master degree in mechanical engineering at the the University Politehnica of Bucharest, Faculty of Mechanical Engineering and Mechatronics Since $2007 \mathrm{Mr}$. Ilie is TTO Manager Relay Centre of Technological Transfer and Consultancy from INCDMTM Bucharest with high expertise in innovation, knowledge transfer and Intellectual Property Rights.

Iulian Ilie has 3 Romanian patents, obtained in collaboration with a team of researchers and his Ph.D. thesis is related to MEMS and MEMS applications. 